\title{
Stroke Prehospital Informed Decision-Making Using EEG Recordings (SPIDER) - CORRIGENDUM
}

Simon Finnigan; ${ }^{1}$ Wayne Loudon; ${ }^{2}$ Andrew Wong; ${ }^{3,4}$ Vivienne Tippett ${ }^{5}$

1. University of Queensland, Centre for https://doi.org/10.1017/S1049023X19001821; Published by Cambridge University Press: 06 May 2019.

Clinical Research (UQCCR), Herston, Australia

2. Queensland Ambulance Service, North Lakes, Australia

3. Royal Brisbane and Women's Hospital, Herston, Australia

4. University of Queensland, St Lucia, Australia

5. Queensland University of Technology, Kelvin Grove, Australia

In the original publication of this abstract, "Stroke Prehospital Informed Decision-Making Using EEG Recordings (SPIDER)," the author order was given incorrectly. ${ }^{1}$ Names should appear as above. The abstract has been corrected.

Reference

1. Finnigan S, Loudon W, Wong A, Tippett V. Stroke prehospital informed decision-making using EEG recordings (SPIDER). Prehosp Disaster Med. 2019;34(Suppl 1):s87.

doi:10.1017/S1049023X19004655 